\title{
Genipin enhances the antitumor effect of elesclomol in A549 lung cancer cells by blocking uncoupling protein-2 and stimulating reactive oxygen species production
}

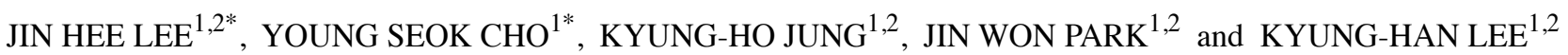 \\ ${ }^{1}$ Department of Nuclear Medicine, Samsung Medical Center, Sungkyunkwan University School of Medicine; \\ ${ }^{2}$ Department of Health Sciences and Technology, Samsung Advanced Institute of Health Sciences and Technology, \\ Sungkyunkwan University, Seoul 06351, Republic of Korea
}

Received January 23, 2020; Accepted September 28, 2020

DOI: $10.3892 / \mathrm{ol} .2020 .12237$

\begin{abstract}
The uncoupling protein-2 (UCP2) serves a role in tumor aggressiveness and anticancer resistance, which is considered to be associated with its ability to attenuate reactive oxygen species (ROS) production. We hypothesized that UCP2 may protect cancer cells from elesclomol-induced cytotoxicity, and that this may be overcome by blocking UCP2 function with genipin. In A549 lung cancer cells that exhibited high UCP2 expression, treatment with elesclomol alone induced limited changes in glucose uptake, ROS production and cell survival. By contrast, both UCP2 knockdown and genipin treatment mildly reduced glucose uptake, increased ROS production and decreased cell survival. Combining genipin and elesclomol further reduced glucose uptake and increased cellular and mitochondrial ROS production. Moreover, co-treatment with genipin and elesclomol reduced the colony forming capacity to $50.6 \pm 7.4 \%$ and the cell survival to $42.0 \pm 3.4 \%$ of that in the control cells (both $\mathrm{P}<0.001$ ). Suppression of cell survival by treatment with elesclomol and genipin was enhanced in the presence of an exogenous ROS inducer and attenuated by a ROS scavenger. The cytotoxic effects of combining genipin and elesclomol were accompanied by reduced mitochondrial membrane potential and occurred through apoptosis as demonstrated by Annexin V assay and increased protein cleavage of PARP and caspase-3. Finally, in an A549 xenograft mouse model, tumor growth was only modestly retarded by
\end{abstract}

Correspondence to: Professor Kyung-Han Lee, Department of Nuclear Medicine, Samsung Medical Center, Sungkyunkwan University School of Medicine, 50 Ilwon-dong, Gangnam, Seoul 06351, Republic of Korea

E-mail:khnm.lee@samsung.com

${ }^{*}$ Contributed equally

Abbreviations: UCP, uncoupling protein; ROS, reactive oxygen species; NAC, N-acetylcysteine; TBHP, tert-butylhydroperoxide; FDG, 18F-fluorodeoxyglucose; PET, positron emission tomography.

Key words: genipin, elesclomol, UCP2, ROS, mitochondria treatment with elesclomol or genipin alone, but was markedly suppressed by combining the two drugs compared with that in the control group $(\mathrm{P}=0.008)$. Therefore, high $\mathrm{UCP} 2$ expression may limit the antitumor effect of elesclomol by attenuating ROS responses, and this may be overcome by co-treatment with genipin; combining elesclomol and genipin may be an effective strategy for treating cancers with high UCP2.

\section{Introduction}

Mitochondria are the main producer of energy (1) and an essential component of redox control in cancer cells (2). Since high levels of reactive oxygen species (ROS) are toxic to cancer cells, mitochondria are a promising target for novel anti-cancer therapeutics. Elesclomol is a first-in-class investigational mitochondria-targeting agent for treating solid tumors (3). Although elesclomol has been reported to induce oxidative stress $(4,5)$, several factors may limit its efficacy for eradicating tumors when used as a single agent. For instance, cancer cells shift energy metabolism from oxidative phosphorylation to glycolysis (6), which helps to prevent excessive ROS accumulation. Cancer cells can also increase tolerance to mitochondria-targeting agents through antioxidant mechanisms (7).

Another potentially major protective mechanism against excessive ROS generation is mitochondrial uncoupling proteins (UCPs), specialized mitochondrial anion transporters that mediate leakage of protons at the inner mitochondrial membrane (8). Among the five types of UCPs in mammalian cells, UCP2, which has been previously linked to obesity and diabetes (9), has gained increasing interest; studies have demonstrated high UCP2 expression in various cancer cells, including those of the breast, ovary, bladder, esophagus, liver, colon, lung and pancreas $(10,11)$. By reducing electron transport chain efficiency, UCP2 increases energy expenditure (9) and reduces ROS generation (8). UCP2 is thus involved in various metabolic disorders (12) and cellular ROS regulation (8). In particular, UCP2-mediated mitochondrial uncoupling exerts protective effects under various stressful conditions, such as excessive production of mitochondrial superoxide ions and aerobic glycolysis stimulation (13). Previous studies have also 
reported that UCP2 serves a role in tumor aggressiveness and anticancer resistance $(14,15)$, which is considered to be associated with its ability to attenuate mitochondrial ROS production $(13,16)$. Thus, blocking UCP2 function may be a viable strategy for eliminating chemotherapy-resistant cancer cells (17).

A promising drug with highly selective inhibitory action against UCP2 activity is a natural compound termed genipin, an aglycone of geniposide present in an extract from Gardenia jasminoides, which has been demonstrated to block UCP2-mediated proton leak in several cell types, such as pancreatic islet cells (9). Previous studies have reported that genipin stimulates ROS generation (18) and induces cytotoxic effects on leukemia and liver cancer cells $(19,20)$. Although combination chemotherapy for cancer has been extensively studied $(21,22)$, the present study focused on combination therapy for cancer cells with poor response to the promising ROS inducer elesclomol and hypothesized the lack of effects of elesclomol on A549 lung cancer cells to UCP2 activity for the first time.

We hypothesized that UCP2 may protect cancer cells from elesclomol cytotoxicity, and that blocking UCP2 function with genipin may enhance the antitumor efficacy of elesclomol. The aim of the present study was to investigate the combined therapeutic effect of genipin and elesclomol, indicating the potential of a cancer inhibition strategy using co-treatment with the UCP2 inhibitor genipin in A549 lung cancer cells, where there are currently limited effects on ROS induction due to the high expression levels of UCP2.

\section{Materials and methods}

Cell culture and reagents. A549 human lung cancer cells from the American Type Culture Collection were maintained in RPMI-1640 medium (Lonza Group, Ltd.) supplemented with $10 \%$ FBS (Serana Europe $\mathrm{GmbH}$ ) and $1 \%$ penicillin/streptomycin (Gibco; Thermo Fisher Scientific, Inc.) at $37^{\circ} \mathrm{C}$ with $5 \% \mathrm{CO}_{2}$ in a humidified atmosphere. Cells were sub-cultured twice a week and used at passages $<15$. The cells were confirmed to be mycoplasma free and authenticated by the Samsung Medical Center institutional research support center. All experiments were performed at cell confluence of $70-80 \%$.

Genipin, $N$-acetylcysteine (NAC) and tert-butylhydroperoxide (TBHP) were obtained from Sigma-Aldrich; Merck KGaA. Treatment concentrations of NAC and TBHP were $10 \mathrm{mM}$ and $200 \mu \mathrm{M}$, respectively, and were co-treated with elesclomol and genipin at $37^{\circ} \mathrm{C}$ for 24 or $48 \mathrm{~h}$. Elesclomol was obtained from Biorbyt. Elesclomol and genipin stocks were dissolved in DMSO at 20 and $250 \mathrm{mM}$, respectively, and further diluted with DMSO. 5-(And-6)-Chloromethyl-2', 7'-dichlorodihydrofluoresscein diacetate acetyl ester (CM-H ${ }_{2}$ DCFDA), MitoSOX Red and Lipofectamine ${ }^{\circledR}$ LTX were obtained from Invitrogen; Thermo Fisher Scientific, Inc. Non-targeting small interfering RNA (siRNA), siRNA targeting UCP2 and the anti- $\beta$-actin antibody (cat. no. 18470) were obtained from Santa Cruz Biotechnology, Inc. Primary anti-UCP2 (cat. no. 89326), anti-cleaved caspase-3 (cat. no. 9661) and anti-PARP (cat. no. 9542), and secondary anti-rabbit (cat. no. 7074) and anti-mouse IgG (cat. no. 7076) antibodies were obtained from Cell Signaling Technology, Inc.
Silencing of UCP2 by siRNA transfection. After the culture medium was changed to that containing 5\% FBS without antibiotics, $80 \%$ confluent A549 cells were transfected with $40 \mathrm{nM}$ UCP2-specific or non-targeted siRNA mixed with Lipofectamine ${ }^{\circledR}$ RNAiMAX (Invitrogen; Thermo Fisher Scientific, Inc.) in opti-MEM (Gibco; Thermo Fisher Scientific, Inc.). At $24 \mathrm{~h}$, the medium was replaced with fresh medium containing $10 \%$ FBS and antibiotics, and the cells were incubated for another $24 \mathrm{~h}$ prior to subsequent experiments.

Clonogenic assay. A549 cells treated with elesclomol and genipin on $100 \mathrm{~mm}$ plates were washed twice with $4^{\circ} \mathrm{C}$ cold PBS, harvested by trypsinization and seeded in 6-well plates at 500 cells/well. After 9 days, the cells were fixed with $100 \%$ methanol at room temperature (RT) for $2 \mathrm{~min}$ and washed twice with cold PBS. The cells were then stained with crystal violet solution (Sigma-Aldrich; Merck KGaA) at RT for $2 \mathrm{~min}$ and washed twice with cold PBS. The number of colonies containing $\geq 50$ cells were finally manually counted in each well using an inverted phase contrast microscopy (magnification, $\mathrm{x} 4$; Olympus Corporation).

Sulforhodamine B (SRB) assay. Cell survival was evaluated by SRB assay in $1 \times 10^{4}$ cells/well seeded overnight in a 96-well plate. Following treatment for 24 and 48 h, A549 cells were fixed in $10 \%$ (wt/vol) trichloroacetic acid at $4^{\circ} \mathrm{C}$ for $2 \mathrm{~h}$. After the cells were stained with SRB dye at RT for $30 \mathrm{~min}$, excess dye was removed by repeated washing with $1 \%(\mathrm{v} / \mathrm{v})$ acetic acid. The protein-bound dye was dissolved in $10 \mathrm{mM}$ Tris base solution, and absorbance at $510 \mathrm{~nm}$ was measured using a VERSA max microplate reader (Molecular Devices, LLC).

Lactate dehydrogenase ( $L D H$ ) activity assay. A549 cells were seeded in a 12-well plate at a density of $2 \times 10^{5}$ cells/well and genipin and elelsclomol treated the following day for $24 \mathrm{~h}$. LDH activity was measured in $500 \mu \mathrm{l}$ cell culture medium for released $\mathrm{LDH}$ and $20 \mu \mathrm{g}$ cell lysate for intracellular LDH using a Cobas c501 assay kit (cat. no. c501; Roche Diagnostics) according to the manufacturer's instructions. Tumor tissues were homogenized with T-PER ${ }^{\mathrm{TM}}$ tissue protein extraction reagent (Thermo Fisher Scientific, Inc.) on ice and centrifuged for $10 \mathrm{~min}$ at $10,000 \mathrm{x} \mathrm{g}$ at $4^{\circ} \mathrm{C}$. Subsequently, $20 \mu \mathrm{g}$ of supernatants were assayed for $\mathrm{LDH}$ activity as above.

Mitochondrial membrane potential (MMP) measurement. A549 cells were seeded in a black 96 -well plate at a density of $4 \times 10^{4}$ cells/well and genipin and elesclomol treated the following day for $24 \mathrm{~h}$. The culture medium was replaced with $100 \mu \mathrm{l}$ phenol red-free RPMI-1640 medium containing 2\% FBS and 500 nM MitoTracker ${ }^{\mathrm{TM}}$ Red FM (Invitrogen; Thermo Fisher Scientific, Inc.), a fluorescent dye that stains the mitochondria of live cells as a function of MMP The cells were incubated for $30 \mathrm{~min}$ at $37^{\circ} \mathrm{C}$ in $5 \% \mathrm{CO}_{2}$ and washed with $100 \mu$ cold PBS per well. The fluorescence in each well was measured using a microplate reader (Mithras LB 940; Titertek-Berthold) using $594 \mathrm{~nm}$ excitation and $642 \mathrm{~nm}$ emission wavelengths.

Annexin $V$ and propidium iodide (PI) flow cytometric analysis. A549 cells at density of $5 \times 10^{5}$ cells $/ \mathrm{ml}$ were 
genipin and elesclomol treated for $24 \mathrm{~h}$, and apoptotic rates were determined using an Annexin V-FITC Apoptosis kit (BD Biosciences) according to the manufacturer's instructions. Annexin $\mathrm{V}$ for early apoptotic cells and propidium iodide (PI) for late apoptotic cells were used. A total of 10,000 cells per sample underwent FACS analysis on a Calibur flow-cytometer (BD Biosciences) using CellQuest software version 5.1 (Becton-Dickinson and Company).

Intracellular ROS measurement. Intracellular ROS levels were quantified using the cell permeant indicator $\mathrm{CM}-\mathrm{H}_{2} \mathrm{DCFDA}$. Briefly, A549 cells were seeded at a density of 4x104 cells/well in a black 96-well plate. After $24 \mathrm{~h}$, the culture medium was changed to $10 \%$ FBS-containing phenol red-free RPMI-1-1640 medium (Gibco; Thermo Fisher Scientific, Inc.) and cells were treated with genipin and elesclomol or transfected with UCP2

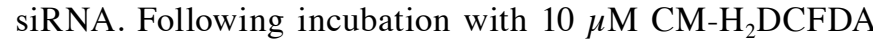
at $37^{\circ} \mathrm{C}$ for $30 \mathrm{~min}$, fluorescence was measured using a GloMax ${ }^{\circledR}-$ Multi Detection System microplate reader (Promega Corporation) at $490 \mathrm{~nm}$ excitation and 510-570 nm emission wavelengths.

Mitochondrial ROS measurement. Mitochondrial ROS was measured using MitoSOX Red with dihydroethidium conjugated to a mitochondria-localization tag. A549 cells were seeded at a density of $4 \times 10^{4}$ cells/well in a black 96 -well plate, and the culture medium was changed the next day to 10\% FBS-containing phenol red-free RPMI-1640 medium. Following genipin and elesclomol treatment, the culture medium was removed, and MitoSOX dye in HBSS buffer (Lonza Group, Ltd.) containing $2 \%$ bovine serum albumin (Bovogen Biologicals Pvt. Ltd.) was added. Following incubation at $37^{\circ} \mathrm{C}$ for $10 \mathrm{~min}$, the buffer was changed to HBSS without dye, and fluorescence was measured on a Mithras LB 940 microplate reader at $510 \mathrm{~nm}$ excitation and $580 \mathrm{~nm}$ emission wavelengths.

Glucose uptake measurement. A549 cells in 24-well plates at a densities of $5 \times 10^{4}$ cells/well were incubated for $40 \mathrm{~min}$ with $100-250 \mathrm{kBq}{ }^{18} \mathrm{~F}$-fluorodeoxyglucose (FDG) (provided by Samsung Medical Centre) added to the culture medium at $37^{\circ} \mathrm{C}$ in $5 \% \mathrm{CO}_{2}$. The cells were rapidly washed twice with cold PBS and lysed with $0.05 \mathrm{~N} \mathrm{NaOH}$, and cell-associated radioactivity was measured on a Wallac high-energy $\gamma$-counter (PerkinElmer, Inc.). Glucose uptake levels were corrected for cell protein content as assessed by Bradford assay.

Immunoblotting. Immunoblotting was performed in A549 cells and tumor tissues lysed with cold PRO-PREP ${ }^{\mathrm{TM}}$ protein extraction solution (iNtRON Biotechnology, Inc.) and T-PER ${ }^{\mathrm{TM}}$ tissue protein extraction reagent (Thermo Fisher Scientific, Inc.), respectively, with protease inhibitor cocktail (Sigma-Aldrich; Merck KGaA). Cell experiments were performed by obtaining cell lysates following 24-h incubation with $250 \mu \mathrm{M}$ genpin and $0.1 \mu \mathrm{M}$ elesclomol Following Bradford assays, $30 \mu \mathrm{g}$ of proteins were separated by $10 \%$ sodium dodecyl sulfate polyacrylamide gel electrophoresis, followed by transfer to a polyvinylidene difluoride membrane. The membrane was blocked with 5\% skim milk in Tris-buffered saline and $0.1 \%$ Tween-20 for $1 \mathrm{~h}$ at room temperature and incubated overnight at $4^{\circ} \mathrm{C}$ with primary antibodies (1:1,000 dilution), followed by $1 \mathrm{~h}$ incubation at room temperature with secondary antibodies (1:2,000 dilution). Immunoreactive protein was detected by chemiluminescence (Pierce ECL plus western blotting substrate; Pierce; Thermo Fisher Scientific, Inc.), and band intensities were quantified using a GS-800 densitometer with Quantity One software version 4.6.6 (Bio-Rad Laboratories, Inc.).

Mouse tumor model and drug treatment. Animal experiments were performed in accordance with the National Institutes of Health Guide for Care and Use of Laboratory Animals and approved by the Institutional Animal Care and Use Committee of Samsung Biomedical Research Institute at Samsung Medical Center (approval no. 20200413001). The tumor model was established in 5-week-old male BALB/c-nude mice weighing 20-25 g (Orient Bio, Inc.) by subcutaneous injection of $5 \times 10^{6}$ A549 cells into the right shoulder. After two weeks, when tumors reached a diameter of $\sim 0.5 \mathrm{~cm}$, the animals were treated with intraperitoneal injections of DMSO control $(n=6), 60 \mathrm{mg} / \mathrm{kg}$ genipin $(\mathrm{n}=6), 30 \mathrm{mg} / \mathrm{kg}$ elesclomol $(\mathrm{n}=7)$ or $60 \mathrm{mg} / \mathrm{kg}$ genipin plus $30 \mathrm{mg} / \mathrm{kg}$ elesclomol $(\mathrm{n}=7)$ three times per week for a total of four weeks. The dosages of genipin and elesclomol were based on previous studies $(23,24)$. The mice were weighed and the tumor size was measured with a caliper before each injection. The experiment was repeated twice for a final total of 6-7 animals per treatment group.

In vivo imaging of tumor FDG uptake. Positron emission tomography (PET) imaging was performed on living tumor-bearing mice on the day following the final drug injection. At $1 \mathrm{~h}$ post-injection with $7.4 \mathrm{MBq}$ of FDG via the tail vein, the animals were anesthetized by isoflurane inhalation, and PET images were acquired on an Inveon micro-PET scanner (Siemens Healthineers). Non-attenuation-corrected tomographic images were analyzed on an Inveon Research Workplace workstation version 4.2 (Siemens Healthineers). Polygonal regions of interest were manually drawn with care to include all tumor mass while excluding adjacent tissue. After PET imaging, the anesthetized mice were sacrificed by cervical dislocation, and the tumor tissues were excised.

Statistical analysis. Data are presented as mean \pm standard deviation or the mean \pm standard error of the mean. Significant differences between groups were analyzed by paired Student's t-test for two groups and one-way ANOVA with Bonferroni post hoc test for multiple groups. $\mathrm{P}<0.05$ was considered to indicate a statistically significant difference.

\section{Results}

Effects of UCP2 knockdown on A549 cell FDG uptake, ROS production and survival. Western blot analysis demonstrated that A549 cells exhibited high protein expression levels of UCP2. Transfection with UCP2-specific siRNA effectively knocked down UCP2 expression to $34.8 \pm 8.0 \%$ of that in the non-targeted siRNA-transfected control cells (Fig. 1A).

Silencing of UCP2 expression with siRNA slightly suppressed FDG uptake to $88.5 \pm 3.3 \%$ of that in control cells (Fig. 1B. ROS production was significantly enhanced by 

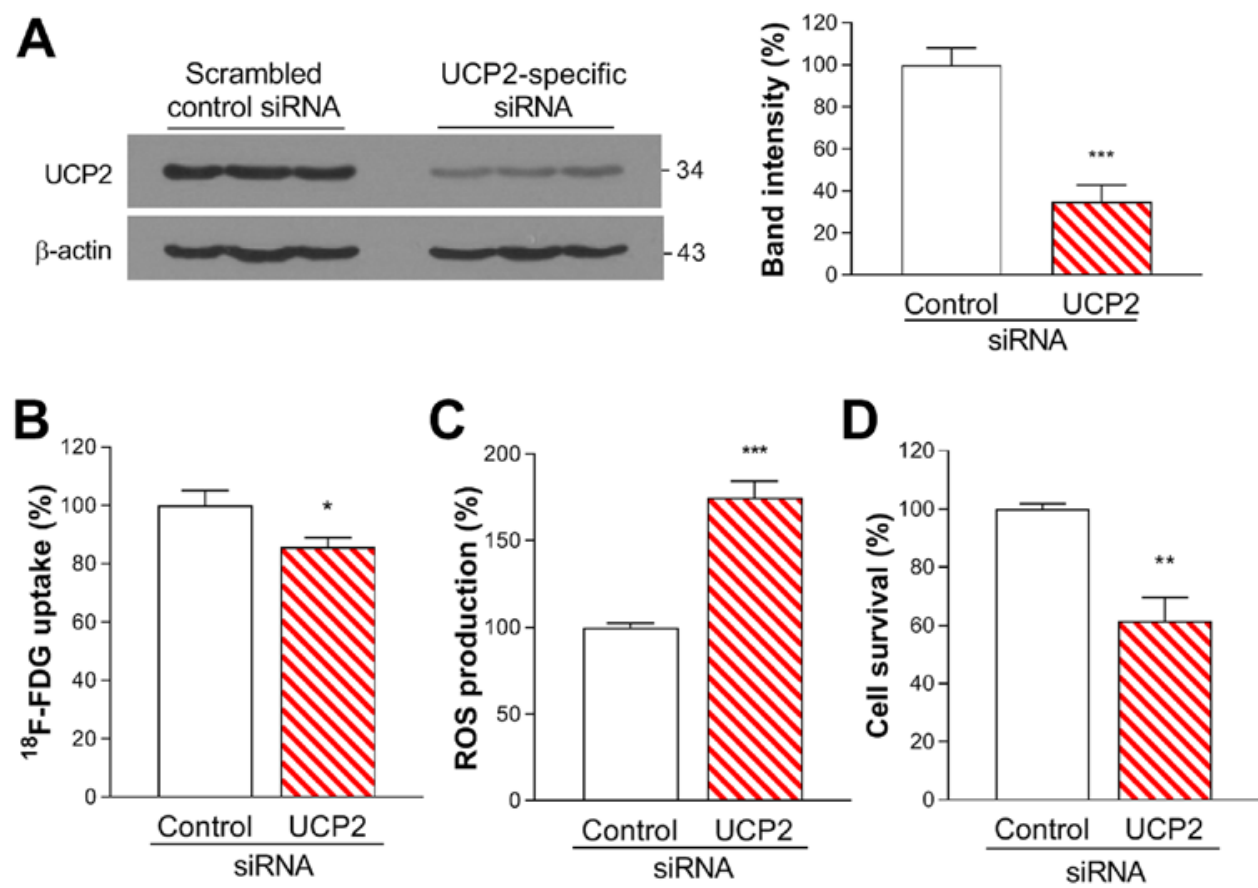

Figure 1. Effects of UCP2 knockdown in A549 lung cancer cells. (A) Western blots of UCP2 protein expression in A549 lung cancer cells transfected with scrambled control or UCP2-specific siRNA. Quantified band intensities were normalized to those of $\beta$-actin. (B) FDG uptake, (C) ROS production and (D) survival of cells transfected with the control or UCP2-specific siRNA. Data are presented as the mean \pm SD of values obtained from triplicate samples of a single representative experiment expressed as percentage values relative to the control siRNA group. ${ }^{*} \mathrm{P}<0.05,{ }^{* * *} \mathrm{P}<0.01$ and ${ }^{* * * *} \mathrm{P}<0.001$ vs. control siRNA. UCP2, uncoupling protein-2; siRNA, small interfering RNA; FDG, ${ }^{18} \mathrm{~F}$-fluorodeoxyglucose; ROS, reactive oxygen species.

UCP2 knockdown to $174.7 \pm 9.6 \%$ of that in controls (Fig. 1C). $\mathrm{SRB}$ assays results revealed that UCP2 silencing significantly decreased A549 cell viability to $60.8 \pm 7.9 \%$ of that in the control group (Fig. 1D).

Effects of genipin and elesclomol on FDG uptake and ROS production. Treatment of A549 cells for $24 \mathrm{~h}$ with $250 \mu \mathrm{M}$ genipin, which blocks UCP2 function without reducing its expression (16), did not affect the protein expression levels of UCP2 (Fig. 2A). FDG uptake was unaffected by 24-h treatment with $0.1 \mu \mathrm{M}$ elesclomol alone, but was significantly suppressed by $250 \mu \mathrm{M}$ genipin alone or by $250 \mu \mathrm{M}$ genipin plus $0.1 \mu \mathrm{M}$ elesclomol to $72.0 \pm 7.1$ and $65.8 \pm 4.6 \%$ of that in the control group, respectively (Fig. 2B).

Intracellular ROS production was also unaffected by $0.1 \mu \mathrm{M}$ elesclomol alone. However, it was increased to $274.9 \pm 1.8 \%$ of that in the control group following 6 -h treatment with $250 \mu \mathrm{M}$ genipin, and was further increased to $404.3 \pm 21.4 \%$ of that in the control group when elesclomol was also present (Fig. 2C). Similarly, mitochondrial ROS production, which was unaffected by elesclomol alone, increased to $169.3 \pm 24.5 \%$ of that in the control group in the cells treated with $250 \mu \mathrm{M}$ genipin and further increased to $256.9 \pm 36 \%$ of that in the control group following co-treatment with genipin and elesclomol (Fig. 2D).

Effects of genipin and elesclomol following UCP2 knockdown. Following UCP2 knockdown, the effects of genipin and elesclomol were more pronounced compared with those in cells transfected with the scrambled control. FDG uptake was decreased to $58.6 \pm 2.6 \%$ of that in the control group by genipin plus elesclomol in the scrambled siRNA-transfected group, and it was further reduced to $42.4 \pm 1.8 \%$ when UCP2 was knocked down (Fig. 2E). Similarly, cytosolic and mitochondrial ROS production levels, which were increased to $252.3 \pm 14.8$ and $186.0 \pm 4.5 \%$ of those in controls by genipin plus elesclomol, respectively, were further increased to $406.8 \pm 35.0$ and $253.2 \pm 27.9 \%$, respectively, when UCP2 was knocked down (Fig. 2F and G).

Effects of genipin and elesclomol on cell clonogenic capacity and survival. The anticancer effects of co-treatment with genipin and elesclomol were analyzed in vitro. Clonogenic assays demonstrated that, when used alone, $0.1 \mu \mathrm{M}$ elesclomol and $250 \mu \mathrm{M}$ genipin exerted limited effects on the colony formation capacity of A549 cells. However, combining the two drugs reduced cell colony numbers to $50.6 \pm 7.4 \%$ of those in the control group following 48-h treatment (Fig. 3A).

Similarly, the results of the SRB assay demonstrated that 48-h treatment with $0.1 \mu \mathrm{M}$ elesclomol or $250 \mu \mathrm{M}$ genipin alone suppressed cell survival to $78.9 \pm 5.2$ and $76.9 \pm 7.1 \%$ of that in the control group, respectively. However, combining the two drugs significantly reduced cell survival to $42.0 \pm 3.4 \%$ of controls (Fig. 3B, middle). Furthermore, LDH release, assessed as a measure of cytotoxicity, was unaffected by 24-h treatment with $0.1 \mu \mathrm{M}$ elesclomol or $250 \mu \mathrm{M}$ genipin alone, but was increased to $128.8 \pm 1.6 \%$ of that in the control group by co-treatment (Fig. 3C), accompanied by decreased intracellular LDH to $63.8 \pm 1.3 \%$ of that in the control group (data not shown).

The role of ROS on the cytotoxic effects of the two drugs was assessed in the presence of the exogenous ROS inducer TBHP and ROS scavenger NAC. The results demonstrated that the cytotoxic effects of genipin, elesclomol and their 
A
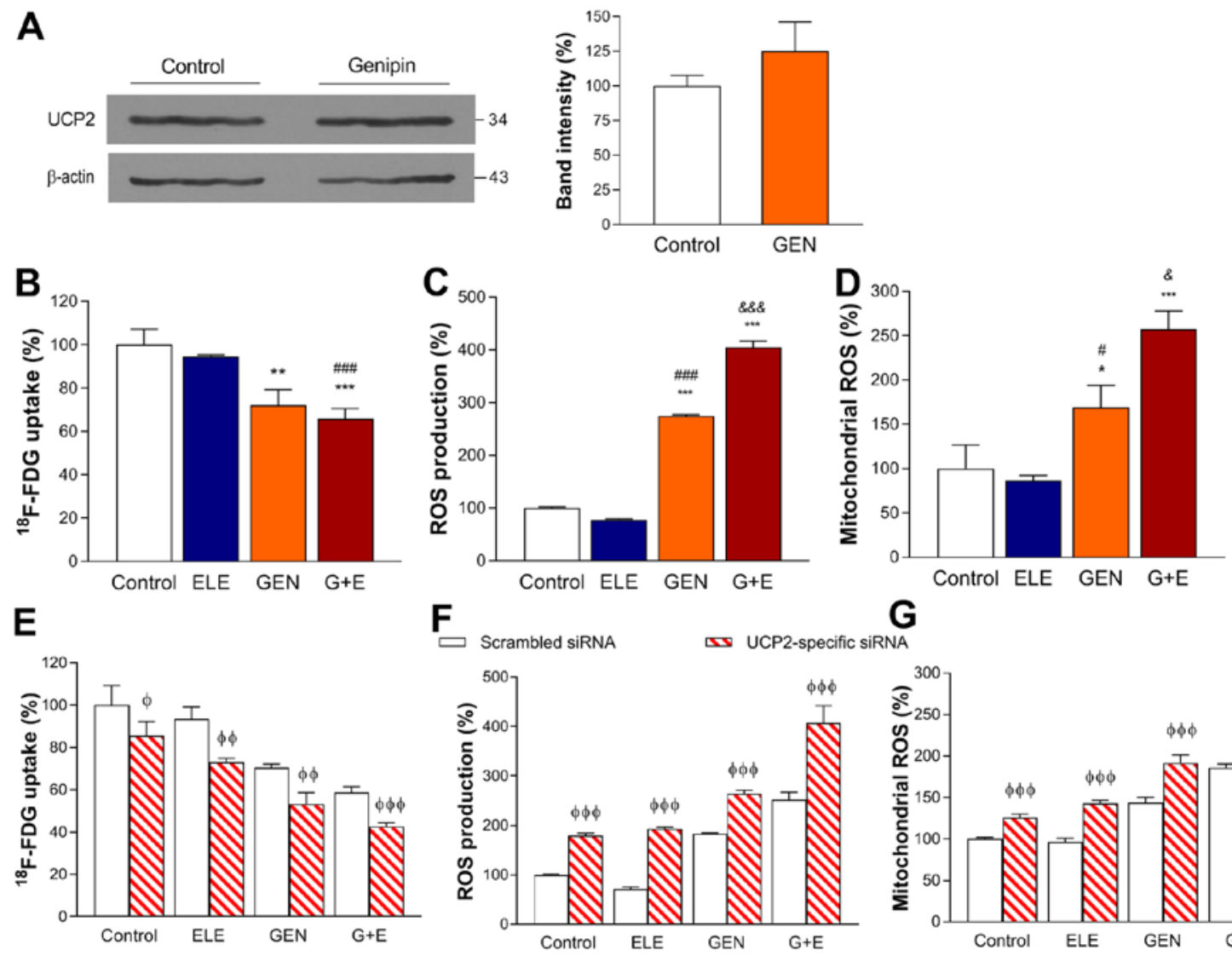

G

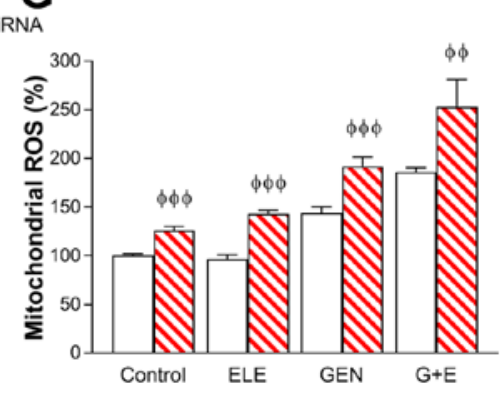

Figure 2. Effects of GEN and ELE treatment on A549 lung cancer cells with or without UCP2 knockdown. (A) Western blots of UCP2 expression in A549 cells following 24-h treatment with $250 \mu \mathrm{M}$ GEN or vehicle (control). Quantified band intensities were normalized to those of $\beta$-actin. (B) FDG uptake, (C) intracellular and (D) mitochondrial ROS production following 24-h treatment with $0.1 \mu \mathrm{M}$ ELE, $250 \mu \mathrm{M}$ GEN, G + E or vehicle (control). (E) FDG uptake, (F) intracellular and (G) mitochondrial ROS production following 48-h ELE, GEN and G + E treatment in cells transfected with scrambled control or $\mathrm{UCP} 2$-specific siRNA. Data are presented as the mean $\pm \mathrm{SD}$ of values obtained from triplicate samples of a single representative experiment expressed as percentage values relative to the control group. ${ }^{*} \mathrm{P}<0.05,{ }^{* *} \mathrm{P}<0.01$ and ${ }^{* * * *} \mathrm{P}<0.001$ vs. control; ${ }^{\#} \mathrm{P}<0.05$, ${ }^{\# \# \#} \mathrm{P}<0.001$ vs. ELE; ${ }^{\&} \mathrm{P}<0.05$, ${ }^{\& \& \&} \mathrm{P}<0.001$ vs. GEN; ${ }^{\phi} \mathrm{P}<0.05$, ${ }^{\phi \phi} \mathrm{P}<0.01$ and ${ }^{\phi} \phi \phi \mathrm{P}<0.001$ vs. scrambled siRNA. GEN, genipin; ELE, elesclomol; G + E, genipin and elesclomol; UCP2, uncoupling protein-2; siRNA, small interfering RNA; FDG, ${ }^{18} \mathrm{~F}$-fluorodeoxyglucose; ROS, reactive oxygen species.

combination were enhanced by TBHP and reduced by NAC; the cell survival in the elesclomol and genipin co-treated cells at $48 \mathrm{~h}$ was decreased to $22.3 \pm 1.9 \%$ of that in the control group by TBHP (Fig. 3D) and was partially recovered to $57.5 \pm 4.8 \%$ of that in the control group by NAC (Fig. 3E).

Effects of genipin and elesclomol on A549 cell apoptosis. The present study next evaluated whether the antitumor effect of combining elesclomol with genipin involved the induction of apoptosis. The results of the MitoTracker Red assay demonstrated that co-treatment with elesclomol and genipin for $24 \mathrm{~h}$ significantly reduced A549 cell MMP to $70.4 \pm 2.5 \%$ of that in the control group (Fig. 4A). This was accompanied by a 6 -fold increase in the percentage of Annexin V-positive apoptotic cells (Fig. 4B). In addition, the co-treatment induced a marked increase in the levels of cleaved PARP to $837.6 \pm 221.9 \%$ and of cleaved caspase -3 to $495.9 \pm 68.6 \%$ of those in untreated controls (Fig. 4C and D).

In vivo antitumor effects of genipin and elesclomol on A549 xenografts in mice. Treatment of A549 tumor xenografts in mice with genipin or elesclomol led to similar magnitudes of modest retardation in tumor growth $\left(280.1 \pm 130.8\right.$ and $310.1 \pm 114.6 \mathrm{~mm}^{3}$ on day 31 , respectively) compared with that in the vehicle-treated control mice (584.2 $\pm 482.5 \mathrm{~mm}^{3}$ on day 31 ; Fig. 5A). By contrast, combination therapy with elesclomol plus genipin resulted in near complete suppression of tumor growth (Fig. 5A); the tumor volume in the co-treatment group $\left(84.0 \pm 27.8 \mathrm{~mm}^{3}\right.$ on day 31$)$ was significantly lower compared with that in the control group No significant differences were observed in animal weight during treatment (Fig. 5A).

$\mathrm{PET} / \mathrm{CT}$ performed after the final treatment revealed significantly smaller tumors with lower FDG uptake in animals co-treated with elesclomol and genipin compared with those in animals treated with control or with elesclomol or genipin alone (Fig. 5B).

Western blots analysis of UCP2 protein expression in the xenograft tumor tissues demonstrated that UCP2 expression levels in the tumors isolated from genipin-treated mice was reduced to $56.4 \pm 12.0 \%$ of those in the tissues from the untreated control group (Fig. 5C). In addition, the LDH levels were lower in the tumor tissue of mice treated with genipin plus elesclomol compared with the control groups (Fig. 5D).

\section{Discussion}

Elesclomol enters cells in a complex with copper, which it transports into the mitochondria to stimulate ROS generation; 

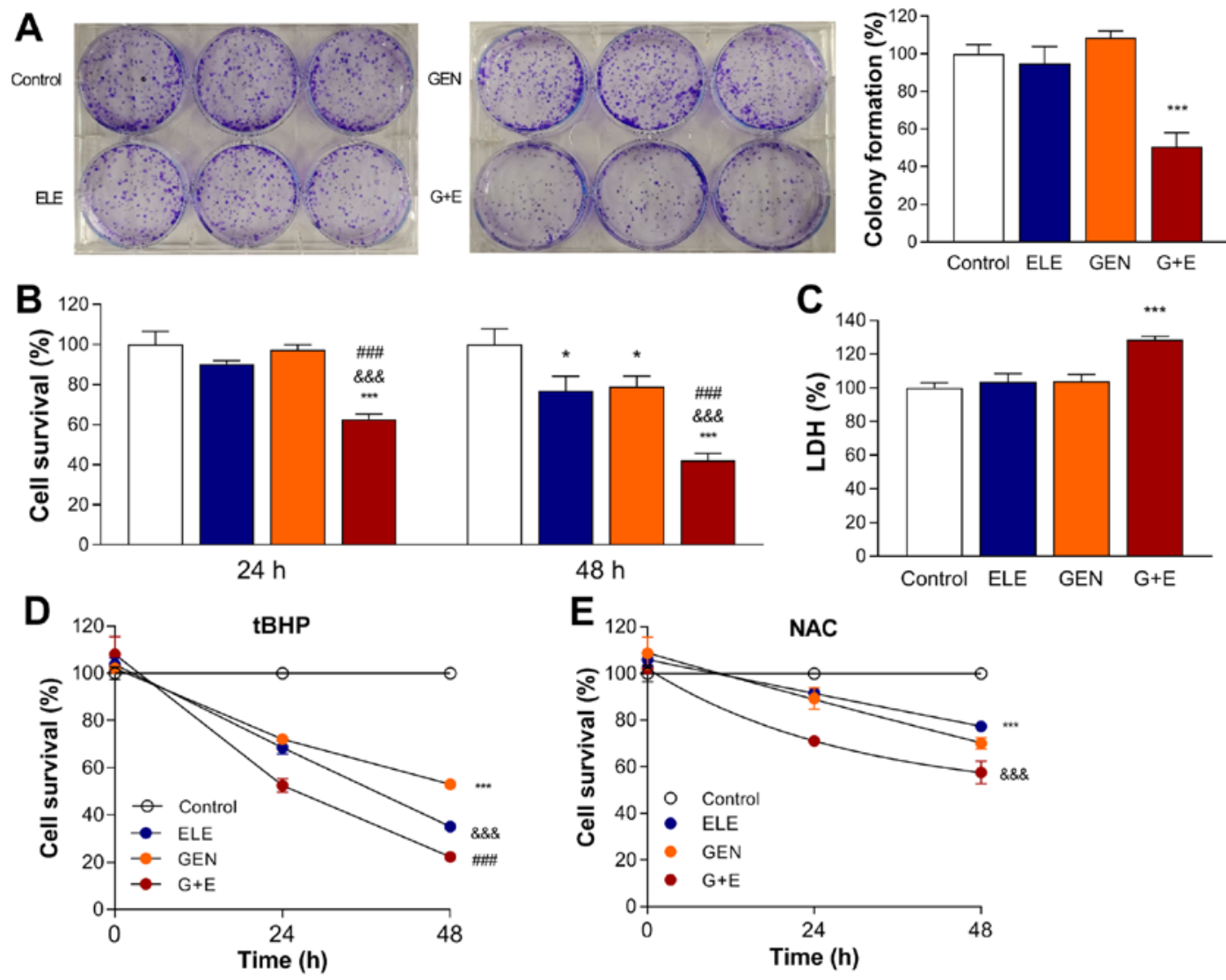

Figure 3. Effects of GEN and ELE on A549 cancer cell viability in vitro. (A) Colony forming capacity of A549 cells following 48-h treatment with $0.1 \mu \mathrm{M}$ ELE, $250 \mu \mathrm{M}$ GEN, G + E or vehicle (control). (B) Cell survival following 24- or 48-h ELE, GEN and G + E treatment and (C) LDH release after 24-h treatment. (D) Effects of $250 \mu \mathrm{M}$ GEN and $0.1 \mu \mathrm{M}$ ELE on A549 cell survival under co-treatment with $200 \mu \mathrm{M}$ ROS inducer TBHP or (E) $10 \mathrm{mM}$ ROS scavenger NAC. Data are presented as the mean \pm SD of the percentage of the control values obtained from triplicate samples of a single representative experiment. ${ }^{*}<0.05$ and ${ }^{* * * *} \mathrm{P}<0.001$ vs. control; ${ }^{\# \# \#} \mathrm{P}<0.001$ vs. ELE; \&\&\& $\mathrm{P}<0.001$ vs. GEN. GEN, genipin; ELE, elesclomol; G + E, genipin and elesclomol; LDH, lactate dehydrogenase; NAC, $N$-acetylcysteine; TBHP, tert-butylhydroperoxide; ROS, reactive oxygen species.

dissociated elesclomol then effluxes out of the cells to repeat mitochondrial copper shuttling and accumulation (25). However, despite its high potential for treating various malignancies including lung cancer (3-5), elesclomol has been limited in increasing ROS and eliminating small and non-small lung cancer cells (26). Although a number of studies have examined the beneficial effects of combining chemotherapeutic drugs for cancer therapy, the present study tested a unique hypothesis that the natural compound genipin may enhance the efficacy of elesclomol to induce ROS generation and promote cell death through the suppression of UCP2 function.

The present study first confirmed that A549 cancer cells exhibited a high level of UCP2 protein expression. In these cells, UCP2 knockdown with a specific siRNA mildly decreased FDG uptake, significantly elevated ROS production and suppressed cell survival compared with those in the control group. When the biological effects of elesclomol and genipin were compared, it was observed that elesclomol monotherapy was unable to significantly affect FDG uptake or ROS production. By contrast, genipin mildly decreased FDG uptake and significantly elevated cellular and mitochondrial ROS production in a manner similar to that obtained by UCP 2 knockdown. Furthermore, the effects were substantially enhanced in cells treated with a combination of elesclomol and genipin. In these experiments, elesclomol and genipin were used at the concentrations of 0.1 and $250 \mu \mathrm{M}$, respectively, which were slightly lower compared with the $0.5 \mu \mathrm{M}$ (4) and $400 \mu \mathrm{M}$ (27) used in previous studies, respectively, to determine their anticancer effects.

Genipin is an inhibitor of UCP2 function rather than expression $(16,20,28-30)$, which is consistent with the result of the present study that the protein levels of UCP2 were not reduced by the genipin treatment. The major role of UCP2 is to inhibit ROS generation (15-19). Inhibition of UCP2 function by genipin may therefore be demonstrated by an increase of ROS production or a reduction of proton leak on oxygen consumption rate analysis. This has been confirmed in various cancer cell types, such as pancreatic cancer cells, breast cancer cells by our group and others $(11,19,20,29-31)$. Thus, the results of the present study that the significantly increased ROS production induced by UCP2 knockdown or genipin may represent the blocking of UCP2 activity-mediated protection against oxidative stress. In addition, the mild reduction of glucose uptake by UCP2 knockdown or by genipin may be explained by the inhibition of the UCP2-mediated glucose expenditure that shifts glucose metabolism toward glycolytic flux $(31,32)$. These effects were more pronounced when genipin treatment was performed in UCP2-knockdown cells, which may indicate a more complete blockage of mitochondrial proton leakage.

When in vitro cytotoxic effects were assessed in the present study, elesclomol alone exhibited limited efficacy in suppressing the colony forming capacity and survival of A549 

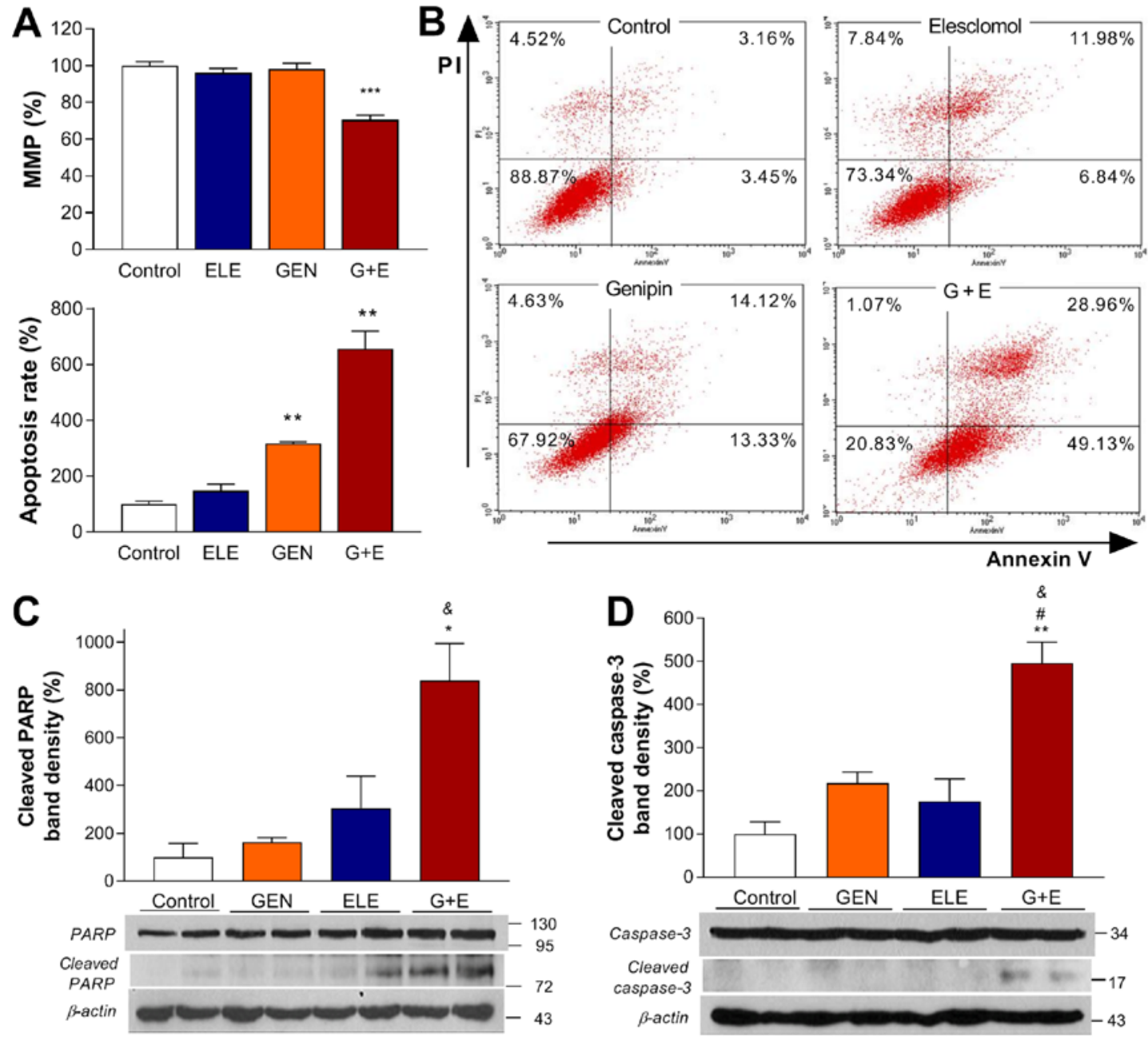

Figure 4. Effects of GEN and ELE on apoptosis in A549 cells. (A) MMP was measured by MitoTracker Red FM, and (B) apoptosis was analyzed using FITC-Annexin V and PI staining following 24-h treatment with $0.1 \mu \mathrm{M}$ ELE, $250 \mu \mathrm{M}$ GEN, G + E or vehicle (control). (C) Western blots of PARP, cleaved PARP, (D) caspase-3 and cleaved caspase-3 following 24-h treatment with ELE, GEN or G + E. Quantified band intensities were normalized to those of $\beta$-actin. Data are presented as the mean $\pm \mathrm{SD}$ of the percentage of the control values obtained from (A and B) triplicate or $(\mathrm{C}$ and $\mathrm{D})$ duplicate samples of a single representative experiment. ${ }^{*} \mathrm{P}<0.05,{ }^{* *} \mathrm{P}<0.01,{ }^{* * *} \mathrm{P}<0.001$ vs. control; ${ }^{*} \mathrm{P}<0.05$ vs. ELE; ${ }^{\circledR} \mathrm{P}<0.05$ vs. GEN. GEN, genipin; ELE, elesclomol; $\mathrm{G}+\mathrm{E}$, genipin and elesclomol; MMP, mitochondrial membrane potential; PARP, poly(ADP-ribose) polymerase.

cells. High UCP2 expression has been previously indicated to attenuate the ROS-stimulatory and cytotoxic effects of paclitaxel (33). Therefore, the limited cytotoxicity of elesclomol observed in the present study may be attributed to the protective action of UCP2 against mitochondrial ROS buildup. This was supported by substantial enhancement by genipin of the ability of elesclomol to suppress A549 cell colony formation and survival, as well as by enhanced cell death in the presence of an ROS donor and suppressed cell death in the presence of a ROS scavenger.

The results of the present study also demonstrated that co-treatment with elesclomol and genipin significantly reduced A549 cell MMP and induced apoptosis, as assessed by Annexin V FACS analysis. This was accompanied by markedly increased levels of the cleaved forms of caspase- 3 and PARP proteins. These results were consistent with the notion that UCP2 protects cancer cells from ROS stress-induced apoptosis (34-36), which occurs through the intrinsic apoptotic pathway (37). Taken together, these results indicated that combining the UCP2 inhibitor genipin enhanced the capacity of elesclomol to induce cancer cell death by activating the intrinsic apoptotic pathway through ROS stimulation and MMP suppression.

Notably, in the present study, the in vivo antitumor effect was also augmented when genipin and elesclomol were used in combination. For these experiments, A549 tumor-bearing mice were treated with $60 \mathrm{mg} / \mathrm{kg}$ genipin, which was within the range of $10-100 \mathrm{mg} / \mathrm{kg}$ previously administered to mice in combination with other drugs $(23,38)$. Elesclomol was administered at a dose of $30 \mathrm{mg} / \mathrm{kg}$, which was in the lower range of the doses previously used for treating tumor-bearing mice (24). These doses were selected to demonstrate that the natural compound genipin may be safely added to enhance the anticancer effects of low-dose elesclomol. The results revealed that elesclomol and genipin therapy alone exhibited modest antitumor efficacies that led to similar retardations of tumor growth. By contrast, combining genipin with elesclomol nearly completely suppressed tumor growth.

Further analysis of the xenograft tumor tissues revealed that the protein levels of UCP2 in tumors isolated from the genipin-treated mice were reduced compared with the control group. This result may indicate the preferential killing of tumor 

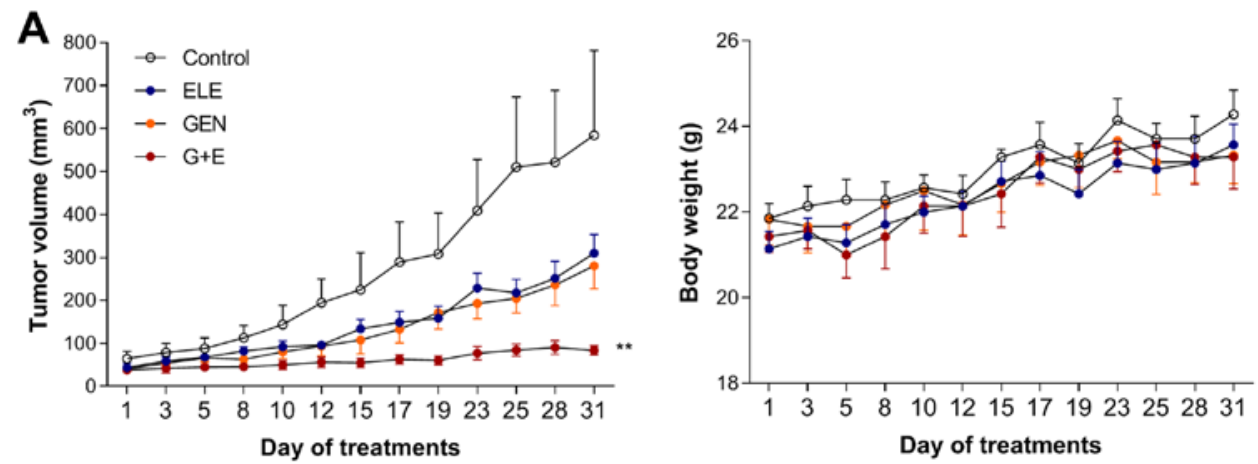

B
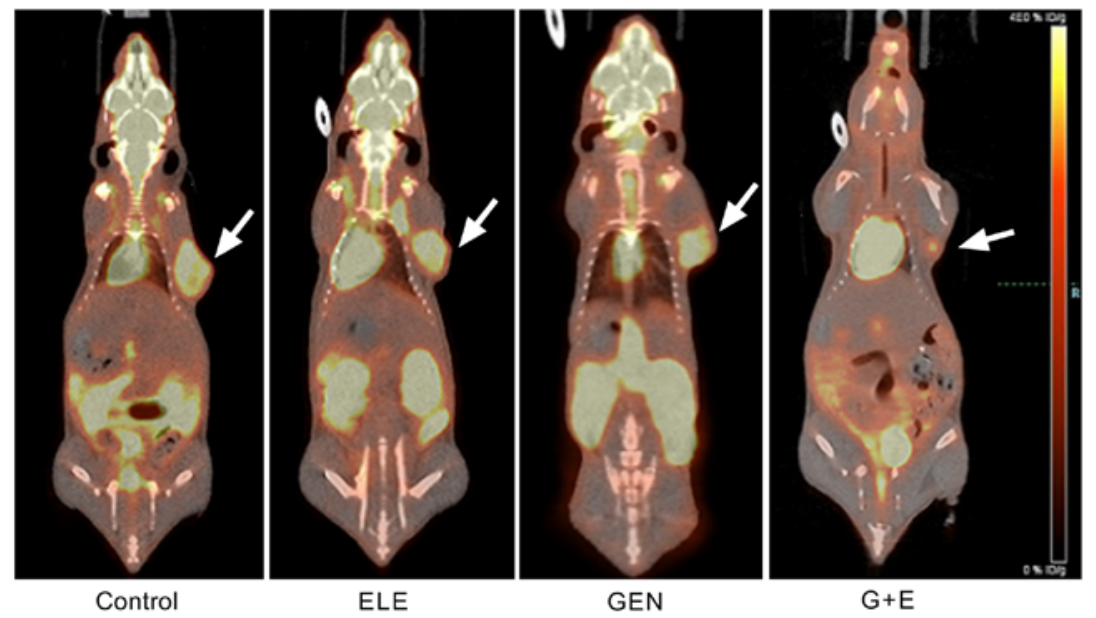

C
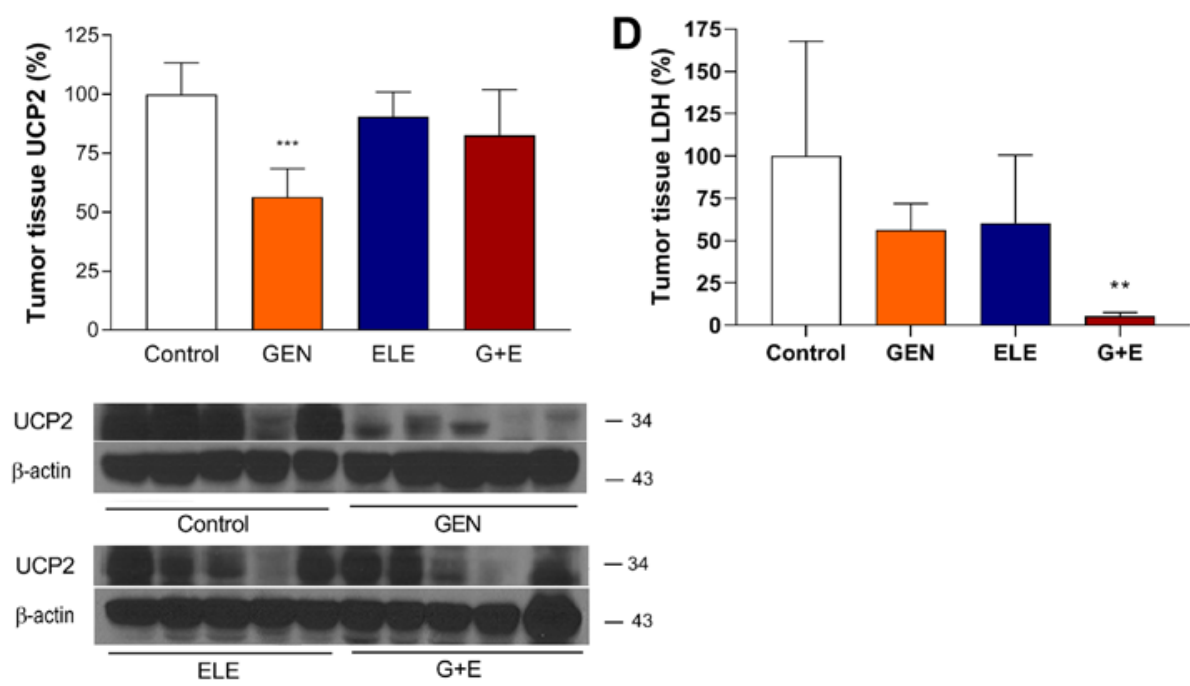

Figure 5. Antitumor effects of GEN and ELE in vivo. (A) Growth of A549 xenograft tumors in mice intraperitoneally injected with DMSO control (n=6), $30 \mathrm{mg} / \mathrm{kg}$ ELE $(\mathrm{n}=7), 60 \mathrm{mg} / \mathrm{kg} \mathrm{GEN}(\mathrm{n}=6)$ or $\mathrm{G}+\mathrm{E}(\mathrm{n}=7)$. The weights of the mice are presented on the right. Data are presented as the mean \pm SEM. ${ }^{\dagger} \mathrm{P}=0.008$ vs. control. (B) Representative coronal positron emission tomography/CT tomographs of mice demonstrating tumor ${ }^{18} \mathrm{~F}$-fluorodeoxyglucose uptake (arrow) after the final treatment. (C) Western blots of UCP2 protein expression and (D) LDH assays in mouse xenograft tumor tissues. Data are presented as the mean $\pm \mathrm{SD}$ of the percentage of the control values obtained from $4-5$ mice per group. ${ }^{* *} \mathrm{P}<0.01$ and ${ }^{* * *} \mathrm{P}<0.001$ vs. control. GEN, genipin; ELE, elesclomol; $\mathrm{G}+\mathrm{E}$, genipin and elesclomol; UCP2, uncoupling protein-2; $\mathrm{LDH}$, lactate dehydrogenase.

cells with greater UCP2 expression by genipin, which led to the remaining tumor tissue containing cells with lower UCP2 levels. Tumor tissue LDH level was reduced in mice treated with genipin plus elesclomol compared with the control group. This finding may indicate that fewer dying cancer cells exist in the reduced tumor tissues after long-term treatment with genipin and elesclomol.

Fig. 6 illustrates our proposed model for the synergistic anticancer effects of elesclomol and genipin. In this model, elesclomol enters cancer cells to induce an increase of mitochondrial oxidative stress, but this effect can be alleviated by UCP2-mediated oxygen consumption uncoupling. UCP2 inhibition with genipin or UCP2 siRNA blocks this protective response and augments the stimulation of ROS production by elesclomol, which in turn triggers apoptosis of the cancer cells.

A limitation of the present study was that the results were restricted to A549 lung cancer cells, which exhibit an efficient UCP2 function, and therefore may not apply to other cancer cell types. It is possible that cancer cells with low UCP2 


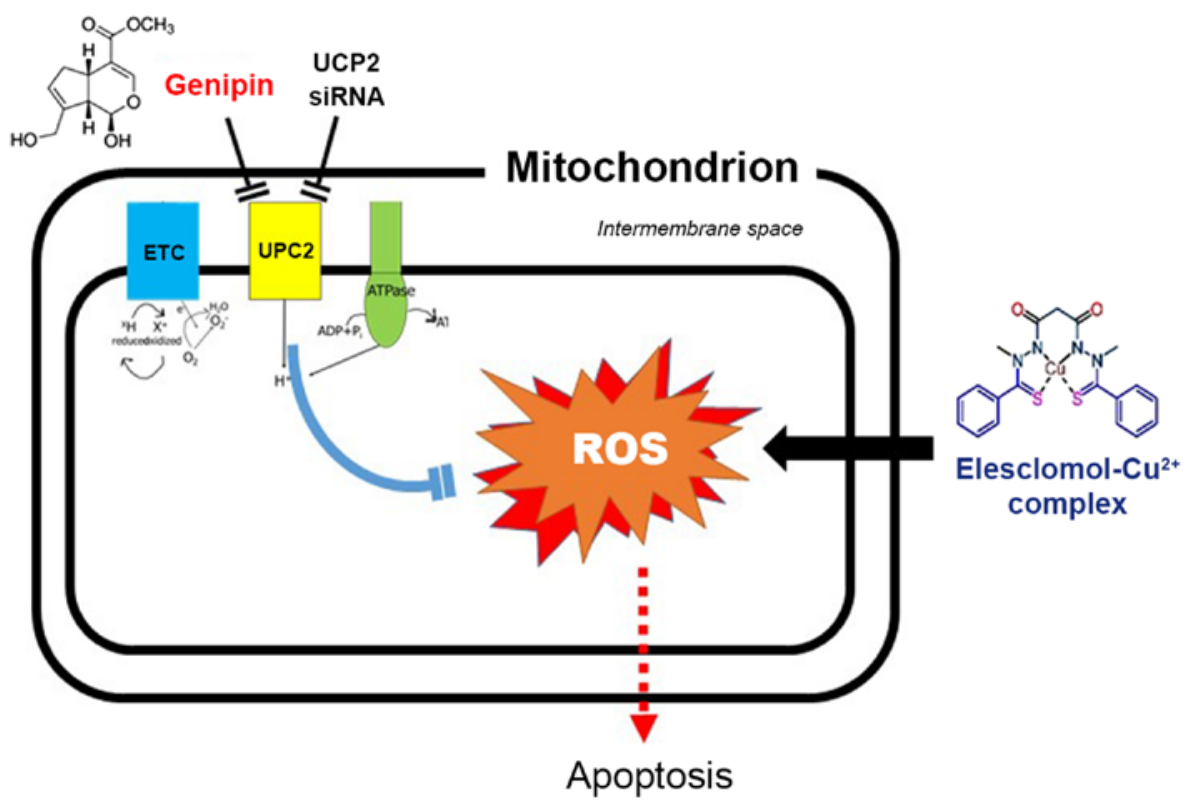

Figure 6. Schematic diagram illustrating the proposed model for the synergistic anticancer effects of elesclomol and genipin. The elesclomol-Cu ${ }^{2+}$ complex enters cancer cells to induce an increase of mitochondrial oxidative stress, which can be reduced by UCP2-mediated uncoupling of oxygen consumption with ATP synthesis. UCP2 inhibition with genipin or UCP2 siRNA blocks this protective response and augments ROS production, which in turn triggers apoptosis. UCP2, uncoupling protein-2; siRNA, small interfering RNA; ROS, reactive oxygen species; ETC, electron transport chain.

expression may respond to elesclomol monotherapy without the need for UCP2 inhibition. By contrast, cancer cells with greater UCP2 activity may benefit even more from the combination of UCP2 inhibitors and elesclomol. In addition, the results of the UCP2 western blots in tumor tissue were limited as only some of the tumor samples were included for analysis. In the animal experiments conducted in the present study, some tumor samples included in the tumor growth data were not included in the UCP2 western data. These issues require validation by further studies.

In conclusion, high UCP2 expression may limit the antitumor effect of elesclomol by attenuating ROS response. This can be overcome by blocking UCP2 function using the natural product genipin, which suppresses glucose uptake, augments mitochondrial ROS production and enhances cytotoxic effects in cancer cells. Therefore, a combination of elesclomol with genipin may be an effective strategy for the treatment of tumors with high UCP expression.

\section{Acknowledgements}

Not applicable.

\section{Funding}

This work was supported by the Basic Science Research Program through the National Research Foundation of Korea funded by the Ministry of Science, ICT and Future Planning (grant nos. NRF-2015R1C1A1A01053454 and NRF-2019R1H1A1035585).

\section{Availability of data and materials}

The datasets used and analyzed during the current study are available from the corresponding author on reasonable request.

\section{Authors' contributions}

KHL, JHL and YSC designed the experiments, analyzed and interpreted the data, and wrote the manuscript. JHL, YSC, KHJ and JWP performed the experiments. All authors read and approved the final manuscript.

\section{Ethics approval and consent to participate}

This study was reviewed and approved by the Institutional Animal Care and Use Committee of Samsung Biomedical Research Institute (SBRI) at Samsung Medical Center (Seoul, Korea). The SBRI is accredited by the Association for Assessment and Accreditation of Laboratory Animal Care International and abides the Institute of Laboratory Animal Resources guide.

\section{Patient consent for publication}

Not applicable.

\section{Competing interests}

The authors declare that they have no competing interests.

\section{References}

1. Chandel NS: Mitochondria as signaling organelles. BMC Biol 12: 34, 2014.

2. Vyas S, Zaganjor E and Haigis MC: Mitochondria and cancer. Cell 166: 555-566, 2016.

3. Berkenblit A, Eder JP Jr, Ryan DP, Seiden MV, Tatsuta N, Sherman ML, Dahl TA, Dezube BJ and Supko JG: Phase I clinical trial of STA-4783 in combination with paclitaxel in patients with refractory solid tumors. Clin Cancer Res 13: 584-590, 2007.

4. Kirshner JR, He S, Balasubramanyam V, Kepros J, Yang CY, Zhang M, Du Z, Barsoum J and Bertin J: Elesclomol induces cancer cell apoptosis through oxidative stress. Mol Cancer Ther 7: 2319-2327, 2008. 
5. Blackman RK, Cheung-Ong K, Gebbia M, Proia DA, He S, Kepros J, Jonneaux A, Marchetti P, Kluza J, Rao PE, et al: Mitochondrial electron transport is the cellular target of the oncology drug elesclomol. PLoS One 7: e29798, 2012.

6. Vander Heiden MG, Cantley LC and Thompson CB: Understanding the Warburg effect: The metabolic requirements of cell proliferation. Science 324: 1029-1033, 2009.

7. Wangpaichitr M, Sullivan EJ, Theodoropoulos G, Wu C, You M, Feun LG, Lampidis TJ, Kuo MT and Savaraj N: The relationship of thioredoxin-1 and cisplatin resistance: Its impact on ROS and oxidative metabolism in lung cancer cells. Mol Cancer Ther 11: 604-615, 2012

8. Arsenijevic D, Onuma H, Pecqueur C, Raimbault S, Manning BS, Miroux B, Couplan E, Alves-Guerra MC, Goubern M, Surwit R, et al: Disruption of the uncoupling protein-2 gene in mice reveals a role in immunity and reactive oxygen species production. Nat Genet 26: 435-439, 2000.

9. Zhang CY,Parton LE, Ye CP, Krauss S, Shen R, Lin CT, Porco JA Jr and Lowell BB: Genipin inhibits UCP2-mediated proton leak and acutely reverses obesity- and high glucose-induced beta cell dysfunction in isolated pancreatic islets. Cell Metab 3: 417-427, 2006.

10. Ayyasamy V, Owens KM, Desouki MM, Liang P, Bakin A, Thangaraj K, Buchsbaum DJ, LoBuglio AF and Singh KK Cellular model of Warburg effect identifies tumor promoting function of UCP2 in breast cancer and its suppression by genipin. PLoS One 6: e24792, 2011.

11. Kuai XY, Ji ZY and Zhang HJ: Mitochondrial uncoupling protein 2 expression in colon cancer and its clinical significance. World J Gastroenterol 16: 5773-5778, 2010.

12. Diano S and Horvath TL: Mitochondrial uncoupling protein 2 (UCP2) in glucose and lipid metabolism. Trends Mol Med 18: $52-58,2012$.

13. Donadelli M, Dando I, Dalla Pozza E and Palmieri M Mitochondrial uncoupling protein 2 and pancreatic cancer: A new potential target therapy. World J Gastroenterol 21: 3232-3238, 2015.

14. Michael A: Pitt: Overexpression of uncoupling protein-2 in cancer: metabolic and heat changes, inhibition and effects on drug resistance. Inflammopharmacol 23: 365-369, 2015

15. Derdak Z, Mark NM, Beldi G, Robson SC, Wands JR and Baffy G: The mitochondrial uncoupling protein-2 promotes chemoresistance in cancer cells. Cancer Res 68: 2813-2819, 2008.

16. Derdák Z, Fülöp P, Sabo E, Tavares R, Berthiaume EP Resnick MB, Paragh G, Wands JR and Baffy G: Enhanced colon tumor induction in uncoupling protein-2 deficient mice is associated with NF-kappaB activation and oxidative stress Carcinogenesis 27: 956-961, 2006.

17. Santandreu FM, Roca $P$ and Oliver J: Uncoupling protein-2 knockdown mediates the cytotoxic effects of cisplatin. Free Radic Biol Med 49: 658-666, 2010.

18. Kim BC, Kim HG, Lee SA, Lim S, Park EH, Kim S-J and Lim CJ: Genipin-induced apoptosis in hepatoma cells is mediated by reactive oxygen species/c-Jun NH2-terminal kinase-dependent activation of mitochondrial pathway. Biochem Pharmacol 70 : 1398-1407, 2005

19. Mailloux RJ, Adjeitey CN and Harper ME: Genipin-induced inhibition of uncoupling protein-2 sensitizes drug-resistant cancer cells to cytotoxic agents. PLoS One 5: e13289, 2010.

20. Khanal T, Kim HG, Choi JH, Do MT, Kong MJ, Kang MJ, Noh K, Yeo HK, Ahn YT, Kang W, et al: Biotransformation of geniposide by human intestinal microflora on cytotoxicity against HepG2 cells. Toxicol Lett 209: 246-254, 2012.

21. Kalemkerian GP: Combination chemotherapy for relapsed small-cell lung cancer. Lancet Oncol 17: 1033-1035, 2016.

22. Li Y, Atkinson K and Zhang T: Combination of chemotherapy and cancer stem cell targeting agents: Preclinical and clinical studies. Cancer Lett 396: 103-109, 2017.

23. Wang N, Zhu M, Tsao SW, Man K, Zhang Z and Feng Y: Up-regulation of TIMP-1 by genipin inhibits MMP-2 activities and suppresses the metastatic potential of human hepatocellular carcinoma. PLoS One 7: e46318, 2012.
24. Gehrmann M: Drug evaluation: STA-4783 - enhancing taxane efficacy by induction of Hsp70. Curr Opin Investig Drugs 7: 574-580, 2006

25. Nagai M, Vo NH, Shin Ogawa L, Chimmanamada D, Inoue T, Chu J, Beaudette-Zlatanova BC, Lu R, Blackman RK, Barsoum $\mathrm{J}$, et al: The oncology drug elesclomol selectively transports copper to the mitochondria to induce oxidative stress in cancer cells. Free Radic Biol Med 52: 2142-2150, 2012.

26. Wangpaichitr M, Wu C, You M, Maher JC, Dinh V, Feun LG and Savaraj N: N',N'-Dimethyl-N',N'-bis(phenylcarbonothioyl) propanedihydrazide (elesclomol) selectively kills cisplatin resistant lung cancer cells through reactive oxygen species (ROS). Cancers (Basel) 1: 23-38, 2009.

27. Feng Q, Cao HL, Xu W, Li XR, Ren YQ and Du LF: Apoptosis induced by genipin in human leukemia K562 cells: Involvement of c-Jun N-terminal kinase in $\mathrm{G}_{2} / \mathrm{M}$ arrest. Acta Pharmacol Sin 32: 519-527, 2011.

28. Valle A, Oliver J and Roca P: Role of uncoupling proteins in cancer. Cancers (Basel) 2: 567-591, 2010.

29. Zhou H, Zhao J and Zhang X: Inhibition of uncoupling protein 2 by genipin reduces insulin-stimulated glucose uptake in 3T3-L1 adipocytes. Arch Biochem Biophys 486: 88-93, 2009.

30. Dando I, Fiorini C, Pozza ED, Padroni C, Costanzo C, Palmieri M and Donadelli M: UCP2 inhibition triggers ROS-dependent nuclear translocation of GAPDH and autophagic cell death in pancreatic adenocarcinoma cells. Biochim Biophys Acta 1833: 672-679, 2013.

31. Cho YS, Lee JH, Jung K-H, Park JW, Moon SH, Choe YS and Lee KH: Molecular mechanism of (18)F-FDG uptake reduction induced by genipin in T47D cancer cell and role of uncoupling protein-2 in cancer cell glucose metabolism. Nucl Med Biol 43 587-592, 2016.

32. Brandi J, Cecconi D, Cordani M, Torrens-Mas M, Pacchiana R, Dalla Pozza E, Butera G, Manfredi M, Marengo E, Oliver J, et al: The antioxidant uncoupling protein 2 stimulates hnRNPA2/B1, GLUT1 and PKM2 expression and sensitizes pancreas cancer cells to glycolysis inhibition. Free Radic Biol Med 101: 305-316, 2016.

33. Su WP, Lo YC, Yan JJ, Liao IC, Tsai PJ, Wang HC, Yeh HH, Lin CC, Chen HH, Lai WW, et al: Mitochondrial uncoupling protein 2 regulates the effects of paclitaxel on Stat 3 activation and cellular survival in lung cancer cells. Carcinogenesis 33: 2065-2075, 2012

34. Horimoto M, Resnick MB, Konkin TA, Routhier J, Wands JR and Baffy G: Expression of uncoupling protein-2 in human colon cancer. Clin Cancer Res 10: 6203-6207, 2004.

35. Harper ME, Antoniou A, Villalobos-Menuey E, Russo A, Trauger R, Vendemelio M, George A, Bartholomew R, Carlo D, Shaikh A, et al: Characterization of a novel metabolic strategy used by drug-resistant tumor cells. FASEB J 16: 1550-1557, 2002

36. Collins P, Jones C, Choudhury S, Damelin L and Hodgson H: Increased expression of uncoupling protein 2 in HepG 2 cells attenuates oxidative damage and apoptosis. Liver Int 25: 880-887, 2005.

37. Wasim L and Chopra M: Synergistic anticancer effect of panobinostat and topoisomerase inhibitors through ROS generation and intrinsic apoptotic pathway induction in cervical cancer cells. Cell Oncol (Dordr) 41: 201-212, 2018.

38. Li CC, Hsiang CY, Lo HY, Pai FT, Wu SL and Ho TY: Genipin inhibits lipopolysaccharide-induced acute systemic inflammation in mice as evidenced by nuclear factor- $\kappa \mathrm{B}$ bioluminescent imaging-guided transcriptomic analysis. Food Chem Toxicol 50: 2978-2986, 2012

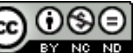

This work is licensed under a Creative Commons Attribution-NonCommercial-NoDerivatives 4.0 International (CC BY-NC-ND 4.0) License. 